Arrays of high- $T_{C}$ Josephson junctions in open millimeter wave resonators

A. M. Klushin, M. He, S. L. Yan, and N. Klein

Citation: Appl. Phys. Lett. 89, 232505 (2006); doi: 10.1063/1.2400401

View online: https://doi.org/10.1063/1.2400401

View Table of Contents: http://aip.scitation.org/toc/apl/89/23

Published by the American Institute of Physics

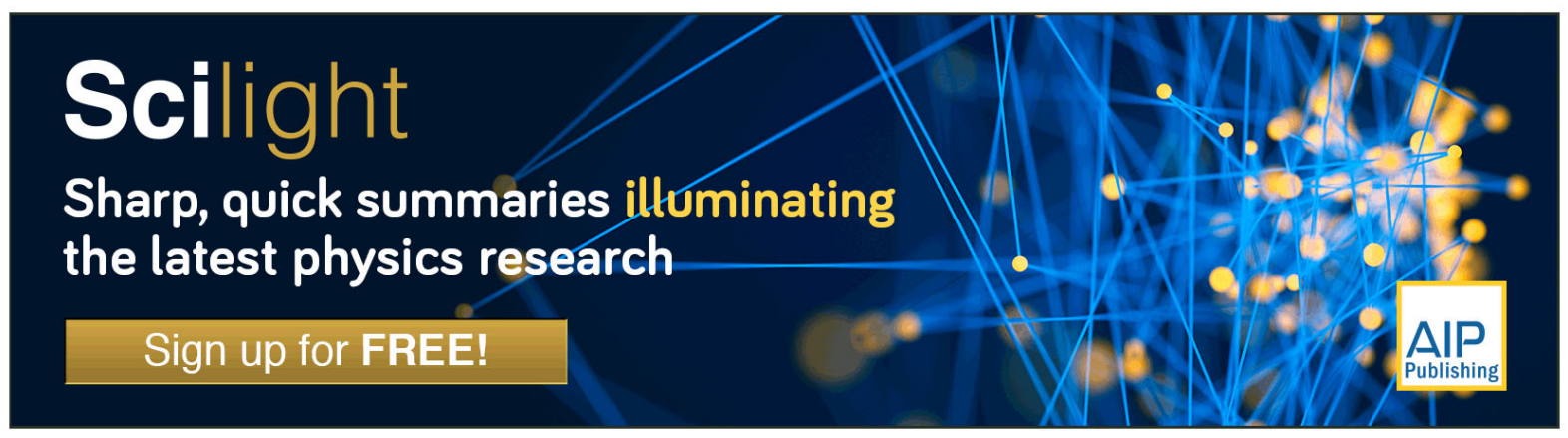




\title{
Arrays of high- $T_{c}$ Josephson junctions in open millimeter wave resonators
}

\author{
A. M. Klushin, ${ }^{\text {a) }}$ M. He, ${ }^{\text {b) }}$ S. L. Yan, ${ }^{\text {b) }}$ and N. Klein \\ Institute for Bio- and Nanosystems and Forschungszentrum Jülich GmbH, D-52425 Jülich, Germany and \\ CNI-Centre of Nanoelectronic Systems for Information Technology, Forschungszentrum Jülich \\ GmbH, D-52425 Jülich, Germany
}

(Received 28 September 2006; accepted 24 October 2006; published online 5 December 2006)

\begin{abstract}
The authors have explored the coupling mechanism of a millimeter wave radiation to bicrystal Josephson junction arrays embedded in a hemispherical Fabry-Pérot resonator. The authors have found that their high-temperature superconductor array can be modeled as a thin film grid polarizer. In agreement with this model, a strong dependence of the coupling of the Josephson junctions on the polarization of the electric filed in the resonator was observed. The authors achieved a maximum Josephson voltage of $28 \mathrm{mV}$ for an array of 182 bicrystal junctions at a temperature of $75 \mathrm{~K}$ and a frequency of $74.4 \mathrm{GHz}$. (C) 2006 American Institute of Physics. [DOI: 10.1063/1.2400401]
\end{abstract}

The challenge of synchronization of Josephson multijunctions circuits was most actively studied due to their application in quantum voltage standards. Typically, straightline series array of niobium Josephson junctions is placed either along a micro-strip-line ${ }^{1}$ or along the center conductor of a coplanar waveguide. ${ }^{2}$ A slot line was also successfully used for irradiation of multijunction arrays. ${ }^{3}$ In all the cases the propagating electromagnetic wave effectively drives the arrays containing up to several thousand junctions.

Special planar thin film based microwave circuits were employed for synchronization of high-temperature superconductor (HTS) bicrystal Josephson junctions for driving frequencies up to $32 \mathrm{GHz}$, and the maximum achieved quantum voltage approached $10 \mathrm{mV} .^{4}$ To further improve the performance of HTS arrays, it is of great importance to raise the maximum driving frequency to about $70 \mathrm{GHz}$ and higher. For [001]-tilt high- $T_{c}$ grain boundary (GB) bicrystal junctions, the typical characteristic frequencies $f_{c}$ exceeded $100 \mathrm{GHz}$ at $77 \mathrm{~K}^{5}$ Moreover, [100]-tilt $\mathrm{YBa}_{2} \mathrm{Cu}_{3} \mathrm{O}_{7-x} \mathrm{~GB}$ junctions with $f_{c}$ up to $0.5 \mathrm{THz}$ at $T=77 \mathrm{~K}$ were explored. ${ }^{6}$ Recently we suggested and successfully realized the method of irradiating arrays of high- $T_{c}$ Josephson junctions by coupling to the resonant modes of a millimeter wave FabryPérot (FP) resonator. ${ }^{7}$

In this letter we describe the mechanism of interaction of Josephson junctions with the millimeter wave electromagnetic field induced in the FP resonator. In particular, we report on our studies about the influence of the series array layout and the polarization of the electric field on the strength of coupling of the resonant electromagnetic field to the junctions.

The FP resonator was arranged as a hemispherical configuration composed of one plane and one spherical mirror. An array of shunted GB Josephson junctions was used as plane mirror. Both the radius of curvature and the diameter of the spherical mirror were $25 \mathrm{~mm}$. Coupling of millimeter wave radiation to the resonator was performed via the open end of a standard waveguide arranged in the center of the spherical mirror. In this case the FP resonator was working in

\footnotetext{
${ }^{\text {a)} E l e c t r o n i c ~ m a i l: ~ a . k l u s h i n @ f z-j u e l i c h . d e ~}$

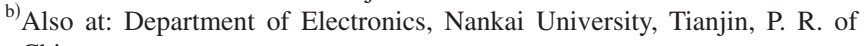
China.
}

the overcoupled mode, i.e., the coupling was stronger than the critical coupling.

The insets in Fig. 1 show the layout of the HTS array circuit. The grain boundary marked as dashed lines is located in the middle of a bicrystal substrate with dimensions of $10 \times 10 \mathrm{~mm}^{2}$. The GB divides the substrate in two equal parts: top and bottom. The metallized parts (light areas in the inserts of Fig. 1) are extended over a length of $4 \mathrm{~mm}$ forming a grid composed of metal stripes of $17 \mu \mathrm{m}$ in width each separated by $5 \mu \mathrm{m}$. The two subgrids below and above the GB are connected in the middle of the substrate with the bridges crossing the GB, forming a series array of 400 junctions with a lateral size of $4 \mathrm{~mm}$. The shunted junctions were fabricated using $\mathrm{Au}-\mathrm{YBa}_{2} \mathrm{Cu}_{3} \mathrm{O}_{7-x}$ bilayers deposited in situ on bicrystal symmetrical yttrium-stabilized zirconium substrates with a misorientation angle of $24^{\circ} .{ }^{8}$ Details of the deposition process and the technology of shunted GB junctions were published previously. ${ }^{9}$

In Fig. 1 the current-voltage $(I-V)$ characteristics of two shunted bicrystal junctions measured at $T=77 \mathrm{~K}$ are shown for different angles $\alpha$ between the grid and the electric field $\boldsymbol{E}$ in the waveguide. The FP resonator was excited at a $\mathrm{TEM}_{00 q}$ resonance (fundamental Gaussian beam) at $f$ $=74.4 \mathrm{GHz}$ at an input power level of $30 \mathrm{~mW}$. For the angle $\alpha=0^{\circ}$, when $\boldsymbol{E}$ is parallel to the grid, coupling is very small. In contrast, by rotation of the waveguide to $\alpha=90^{\circ}$, such that $\boldsymbol{E}$ is perpendicular to the grid, a strong suppression of the

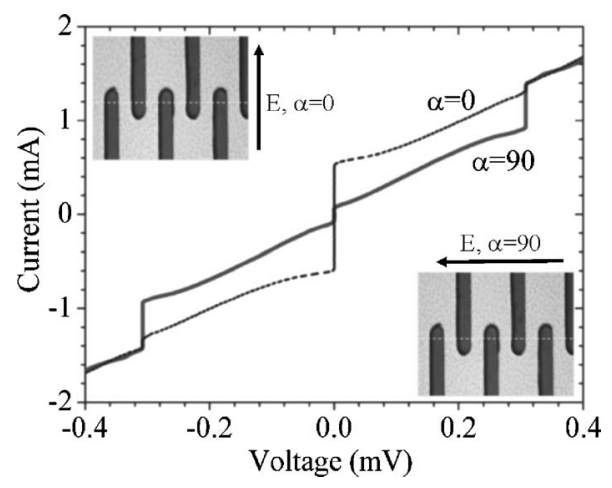

FIG. 1. I- $V$ characteristics of two bicrystal junctions array for two angles $\alpha$. The grain boundary $(\mathrm{GB})$ is shown as a white dashed line. 


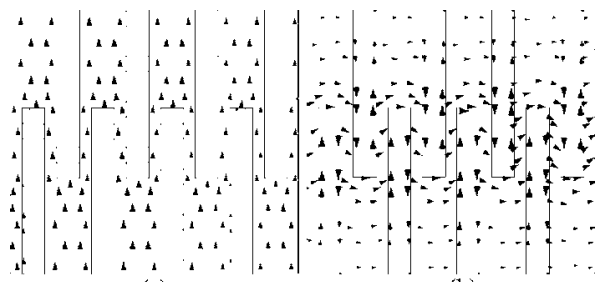

(a)

(b)

FIG. 2. Simulation of the current distribution in the thin film stripe grid when the electric field is parallel (a) and perpendicular (b) to the thin film stripes.

critical current $I_{c}$ and a maximum height of the first Shapiro step $\Delta I_{1}$ were observed.

To explain such a dependence, we consider the analogy between our circuit and a wire grid polarizer. ${ }^{10}$ A plane electromagnetic wave with the electric field aligned parallel to the wires induces the movement of electrons along the wires such that the wave is reflected backwards along the incident beam. We have performed numerical field simulation of our structure with CST MICROWAVE STUDIO. ${ }^{11}$ Within the suggested model, plane wave excitations are employed to calculate the surface current distribution in the metal thin film structure. These calculations have been performed for different orientation angles and frequencies. The results of simulations confirm our assumption, as shown in Fig. 2(a). AC currents flow uniformly along the thin film metal stripes resulting in a strong reflection and negligible coupling to the Josephson junctions.

On the other hand, for the electromagnetic waves with the electric field perpendicular to the stripes, the electrons cannot move very far across the width of each stripe. Therefore, only a small amount of energy is lost by reflection, and the incident wave is able to travel through the grid. Since the electric field components parallel to the stripes are strongly reflected, the transmitted wave has an electric field purely in the direction perpendicular to the stripe. In this case the ac currents concentrate inside the bridges, which provides a strong ac bias to the array, as shown in Fig. 2(b). We suppose that coupling can be further increased by optimizing the substrate thickness.

Figure 3 shows the dependence of the critical current $I_{c}$ and $\Delta I_{1}$ on the angle from $0^{\circ}$ to $90^{\circ}$. The experimental results were fitted by the empirical dependence $I_{c}, \Delta I_{1}$ $\sim \cos (\alpha)$, confirming our suggested model.

Furthermore, we investigated Shapiro steps measured for different subarrays containing 8, 16, 32, 64, 101, 115, 131, 163, and 182 junctions, respectively. Figure 4 shows the

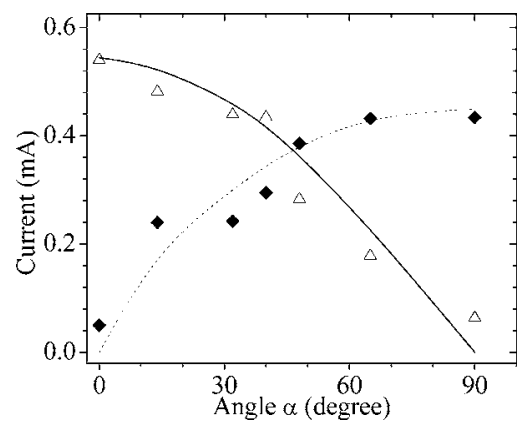

FIG. 3. Experimental dependencies of the critical current $(\triangle)$ and the first Shapiro step $(\diamond)$ on the angle $\alpha$. The straight and dashed lines represent empirical dependence of $I_{c}, \Delta I_{1} \sim \cos (\alpha)$.

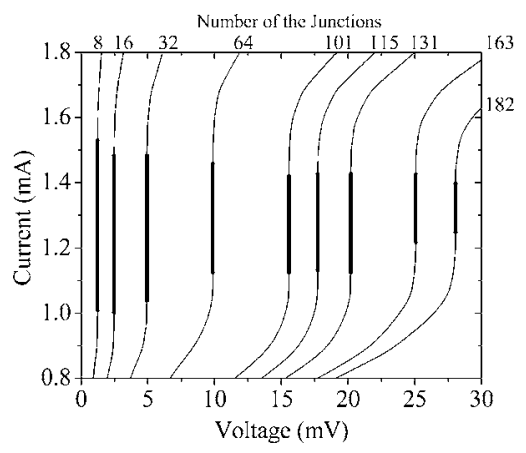

FIG. 4. $I$ - $V$ characteristics for nine subarrays from 1.2 to $28 \mathrm{mV}$. All $I-V$ 's were measured with the same power of $30 \mathrm{~mW}$ at $74.4 \mathrm{GHz}$. The bold lines demonstrate the first Shapiro step height measured with resolution better than $1 \mu \mathrm{V}$.

$I-V$ characteristics measured at $75 \mathrm{~K}$ with irradiation frequency of $f=74.4 \mathrm{GHz}$. The Josephson junctions in the arrays showed the average critical current of $I_{c}=0.7 \mathrm{~mA}$, and the average resistance of one shunted junction was about $0.14 \Omega$. The resulting characteristic frequency $f_{c}$ was about $50 \mathrm{GHz}$. The bold lines in Fig. 4 indicate the height $\Delta I_{1}$ of the first Shapiro step for different HTS subarrays. The step heights were measured with resolution better than $1 \mu \mathrm{V}$. Precision measurements revealed that we achieved about $20 \mathrm{mV}$ on the subarray containing 131 junctions with $\Delta I_{1}$ $\cong 0.3 \mathrm{~mA}$ and about $25 \mathrm{mV}$ on the subarray containing 163 junctions with $\Delta I_{1} \cong 0.25 \mathrm{~mA}$.

The maximum number of junctions being synchronized was equal to 182 . In this subarray a step height $\Delta I_{1}$ equal to $0.15 \mathrm{~mA}$ at $28 \mathrm{mV}$ was observed. Since the width of the junctions was $6 \mu \mathrm{m}$ and the distance between the junctions was $5 \mu \mathrm{m}, 182$ junctions were spaced over a distance of $2 \mathrm{~mm}$. We suppose that the limitation is caused by the nonuniformity of the field determined by the width of the Gaussian beam in the fundamental mode of the FP resonator. This is no fundamental limitation, since the width of the Gaussian beam can be increased by increasing the radius of curvature and diameter of the spherical mirror.

In summary, we have investigated the coupling mechanism of millimeter wave radiation to Josephson junction arrays embedded in a wire grid polarizer structure, which is arranged in a hemispherical FP resonator. A strong dependence of the coupling to the Josephson junctions on the polarization of the electric field in the resonator was measured. These results were confirmed by numerical simulations. Arrays with 182 junctions were synchronized and a maximum quantum Josephson voltage equal to $28 \mathrm{mV}$ was measured at a temperature of $75 \mathrm{~K}$ for the first time. Subarrays containing smaller number of junctions were also synchronized at the same frequency. Our results showed that such circuits are challenging for application as a programmable voltage standard. It is important to note that our quasioptical coupling method can be extended up to terahertz frequencies. Here, our approach appears very promising for the realization of detector arrays. Finally, our approach could be useful for irradiation of large arrays of niobium Josephson junctions as well. In this case, a substantial simplification of the technology of niobium arrays and an increase of the irradiation frequency could be achievable.

${ }^{1}$ J. Niemeyer, J. H. Hinken, and R. L. Kautz, Appl. Phys. Lett. 45, 478 (1984). 
${ }^{2}$ S. P. Benz, Appl. Phys. Lett. 67, 2714 (1995).

${ }^{3}$ M. Schubert, T. May, G. Wende, L. Fritzsch, and H.-G. Meyer, Appl. Phys. Lett. 79, 1009 (2001).

${ }^{4}$ A. M. Klushin, R. Behr, K. Numssen, M. Siegel, and J. Niemeyer, Appl. Phys. Lett. 80, 1972 (2002).

${ }^{5}$ H. Hilgenkamp and J. Mannhart, Rev. Mod. Phys. 74, 485 (2002).

${ }^{6}$ Y. Y. Divin, U. Poppe, C. L. Jia, P. M. Shadrin, and K. Urban, Physica C 372-376, 115 (2002).

${ }^{7}$ A. M. Klushin, D. M. Druzhnov, and N. Klein, J. Phys.: Conf. Ser. 43,
1155 (2006).

${ }^{8}$ Theva GmbH, http://www.theva.de

${ }^{9}$ A. M. Klushin, C. Weber, M. Darula, R. Semerad, W. Prusseit, H. Kohlstedt, and A. I. Braginski, Supercond. Sci. Technol. 11, 609 (1998).

${ }^{10}$ P. F. Goldsmith, Quasioptical Systems: Gaussian Beam, Quasioptical Propagation and Applications, IEEE/Hall Series on Microwave Technology and Techniques (IEEE, New York, 1997), pp. 192-196.

${ }^{11}$ CST Microwave Studio, http://www.cst.com 